\title{
EDITORIALE
}

\section{Le imprese alimentari e la protezione del benessere animale nelle società multiculturali}

Irene Canfora*

La questione del bilanciamento nella definizione di valori fondamentali, garantiti dai testi costituzionali e dai trattati europei, è una costante della riflessione giuridica. Essa trova sempre nuove esigenze di confronto, quando i valori tutelati e i principi fondamentali espressi dalla società si evolvono e si raffinano in ragione delle sensibilità maturate nei rapporti sociali, come accade per effetto della globalizzazione.

La prospettiva che si vuole indagare nei contributi che compaiono in questo fascicolo tocca alcuni aspetti che incidono sulla regolazione delle attività delle imprese della filiera agroalimentare, con riguardo all'esigenza di conciliare la protezione degli animali da reddito con le espressioni del pluralismo culturale e la tutela della libertà religiosa nel territorio europeo, alla luce dei principi generali definiti nei Trattati, dell'ordinamento europeo e degli ordinamenti statuali.

Il tema, che potremmo ricondurre alla dimensione dello "sviluppo sostenibile” ed alla pluralità delle sue articolazioni - dalla protezione dell'ambiente, al miglioramento delle condizioni sociali di vita e di benessere della popolazione, all'inclusione delle diverse espressioni del pluralismo culturale e religioso - impone una riflessione sulla coesistenza dei valori tutelati e, di volta in volta, pone l'esigenza di conciliarne la combinazione tra loro, affinché non siano pretermesse istanze e interessi emergenti nella società civile. Si tratta di individuare un punto di equilibrio tra interessi e valori, che appartengono alle più recenti istanze della "modernità" e che si collocano su piani spesso paralleli: laddove essi si intersecano - e ciò avviene in particolare nell'ambito delle attività produttive degli alimenti di origine animale e della loro immissione nel mercato degli alimenti - occorre indivi-

*Università degli Studi di Bari Aldo Moro; irene.canfora@uniba.it

Agricoltura - Istituzioni - Mercati, n. 1/2018, ISSN 1828-194X, ISSNe 1971-8373

DOI: 10.3280/AIM2018-001001 
duare una chiave di lettura interpretativa che garantisca il raggiungimento degli obiettivi posti dall'ordinamento giuridico.

Quanto al rapporto tra normativa sul benessere animale e tutela dell'espressione delle religioni, il primo aspetto da prendere in considerazione è dato dalla efficacia dell'enunciazione dei principi e dalla scala di valori definita dall'ordinamento giuridico.

Il secondo tema, anch'esso affrontato nei saggi che seguono, riguarda la definizione di una normativa sul processo di produzione e sulla comunicazione al consumatore adeguata a dare attuazione a tali principi.

Quanto all'affermazione dei valori e alla "scala” delle priorità, si registra una scelta di fondo nelle disposizioni del Trattato, nel fissare valori unitari, che sanciscono, quale parametro regolativo delle "politiche dell'Unione nei settori dell'agricoltura, della pesca, dei trasporti, del mercato interno, della ricerca e sviluppo tecnologico e dello spazio dell'ordinamento giuridico europeo" una considerazione degli animali come "esseri senzienti” (all'art. 13 Tfue), purché ciò avvenga nel rispetto delle consuetudini degli Stati, delle tradizioni culturali e del rispetto dei riti religiosi. Tale affermazione incontra due limiti significativi: in primo luogo, essa lega la sua operatività alla concreta attuazione delle politiche, sminuendone per certi versi l'impatto in termini di valori e di affermazione di principi aventi carattere generale nell'ordinamento; in secondo luogo affida la soluzione del potenziale conflitto tra i due valori (protezione del benessere animale e rispetto delle tradizioni culturali e religiose) ad una regolazione che, per il secondo, è decentrata e diffusa - dunque non legata all'adozione di norme vincolanti, ma piuttosto fondata sul richiamo alle consuetudini e alle tradizioni culturali, che possono giustificare tanto un fondamento normativo nazionale, quanto un approccio di soft law o anche la mancanza di una regolazione.

In sostanza, nella formulazione della norma, la considerazione degli aspetti culturali, ma soprattutto religiosi, che possono interferire con il rispetto delle norme sul benessere animale, appare quasi come un limite "esterno", la cui implementazione è affidata a fonti eteronome, perché legate alle sensibilità di tradizioni non necessariamente codificate o codificabili.

Conseguentemente, mentre la normativa europea di settore ha adeguato le regole di produzione ai parametri volti a garantire il benessere animale, altra questione ha riguardato la previsione di norme specifiche, in parte derogatorie, dirette a rispondere, già nell'ambito degli atti normativi di fonte europea, alle istanze provenienti da una società multiculturale e legate al rispetto di speciali dettami religiosi.

Infatti, quanto al secondo livello di regolazione, consistente nella definizione e nell'attuazione delle politiche europee (in particolare, per l'angolo 
visuale preso in considerazione dai saggi raccolti in questo fascicolo, quello delle produzioni agroalimentari), alla luce dei regolamenti europei, gli aspetti del benessere animale sono divenuti ormai parte integrante della disciplina giuridica nell'ambito della filiera agroalimentare - dove esiste già uno "statuto" di regole applicabili alle attività delle imprese, che hanno ad oggetto una "universalità di animali" in quanto fattori di produzione: si pensi alle regole dell'allevamento, del trasporto, della macellazione - ma anche alla loro correlazione con le norme di finanziamento della politica agricola comune.

Questa strada è ampiamente consolidata: nel settore agricolo, le regole della condizionalità, presupposto per l'accesso alle misure di sostegno per i produttori agricoli, includono sin dalla loro introduzione le norme sul benessere animale negli allevamenti; le norme igienico-sanitarie sulla produzione degli alimenti di origine animale prevedono disposizioni relative al benessere nella produzione; regolamenti europei disciplinano dettagliatamente le norme sulla protezione degli animali durante il trasporto e le norme sulla macellazione degli animali da reddito. Infine, ancora più recentemente, il regolamento 2017/625 nel definire una disciplina organica dei controlli ufficiali sugli alimenti e sui mangimi, dispone l'istituzione dei centri di riferimento per il benessere degli animali.

A fronte di un appartato regolativo così strutturato, il punto di frizione del sistema, si registra rispetto all'altro valore contemplato dalla disposizione dell'art 13 del Tfue, che fondamentalmente legittima la presenza di norme appartenenti a un livello regolativo differente, quello religioso, che si può trovare a confliggere con le regole tecniche dettate per ragioni sanitarie e di protezione del benessere animale, definite quali standard europei per l'allevamento degli animali e per la presentazione degli alimenti.

Tale situazione si amplifica con la trasformazione di un mercato in cui cresce il multiculturalismo, per ragioni etiche, culturali e religiose, frutto delle migrazioni e dei mutamenti negli approcci culturali con una incidenza diretta sull'evoluzione dei consumi della popolazione europea. Un mercato ormai largamente diversificato nelle caratteristiche dei prodotti alimentari a fronte di una pluralità di figure eterogenee di consumatori espressione di scelte culturali etiche o religiose differenti.

In questo quadro, le forme di comunicazione relative alle caratteristiche del prodotto rappresentano un aspetto fondamentale per la trasparenza delle scelte dei consumatori.

Assume allora rilievo la trasparenza dei contenuti normativi che l'etichettatura trasferisce al consumatore. Solo la certezza dei contenuti normativi e della trasparenza nelle scelte del bilanciamento tra opposti interessi, 
da parte dal legislatore, può garantire che la protezione del consumatore finale e il rispetto delle scelte alimentari risponda a un suo legittimo affidamento.

Si pone dunque ancora una volta l'interrogativo, se e quanto gli strumenti regolativi di soft law siano efficaci, non solo al fine di garantire l'effettiva trasparenza e la non ingannevolezza dell'informazione, ma al fine di realizzare quel bilanciamento tra valori che il Trattato dichiara in via di principio, affidandone poi l'attuazione a strumenti regolativi non necessariamente di stampo legislativo. La questione torna al tema della trasparenza delle informazioni collegate alle certificazioni, quando esse non siano regolate da precisi parametri normativi, ma rimesse a soggetti privati che ne definiscono le caratteristiche oggetto della certificazione, pur entro i confini stabiliti dai parametri della correttezza della concorrenza e della veridicità delle informazioni.

In verità, è proprio la chiara definizione delle regole di processo che meriterebbe l'attenzione degli organi europei, anche in considerazione di potenziali conflitti tra i diversi "valori" che rispecchiano le scelte dei consumatori di alimenti. Valori che, in certi casi, finiscono per sovrapporsi, proprio per effetto dell'evoluzione delle abitudini che un mondo multiculturale e globalizzato tende ad avvicinare.

E il caso di richiamare, a tale proposito, la decisione della Corte di Giustizia che si è trovata a dirimere un conflitto tra diversi segmenti dello stesso "mercato alimentare" - quello delle produzioni ottenute con il metodo biologico, strutturato in dettagliatissime regole tecniche (ma non fino al punto da occuparsi della macellazione rituale) e quello delle carni macellate secondo regole prescritte da riti religiosi. La questione riguardava l'uso dell'etichettatura biologica per alimenti che fossero certificati anche come kasher o halal, ammettendo quindi una intersezione tra due diversi sistemi di regolazione del processo di produzione.

La Corte di Giustizia, con sentenza del 26 febbraio 2019, in causa C497/17, ha negato l'uso del logo "biologico" per le carni macellate con metodi prescritti da riti religiosi, sulla base dell'applicazione del principio generale previsto dal regolamento sulla produzione biologica, consistente nel "mantenere un elevato livello di benessere degli animali".

Invero, così come tutti i principi enunciati dal regolamento, esso si traduce poi nella conseguente esplicita previsione di norme tecniche, disposte nel regolamento di attuazione.

Non può negarsi che la decisione citata susciti qualche perplessità, in assenza di violazione espressa delle norme tecniche - come, peraltro, aveva sottolineato l'avvocato generale Niels Wahl, nelle sue conclusioni del 20 
settembre 2018, giungendo ad una soluzione opposta a quella adottata dalla Corte. Ciononostante, la Corte propende per l'esigenza di "tutelare e giustificare la fiducia del consumatore nei prodotti etichettati come biologici", dovendosi pertanto "vigilare affinché ai consumatori sia garantito che i prodotti che recano il logo biologico dell'Unione Europea siano effettivamente ottenuti nel rispetto delle norme più elevate, segnatamente in materia di benessere animale". Afferma la Corte che "i metodi particolari di macellazione prescritti da riti religiosi, che sono eseguiti senza previo stordimento, non equivalgono, in termini di garanzia di un livello elevato di benessere degli animali al momento del loro abbattimento, al metodo della macellazione con stordimento previo".

Il dilemma si risolve sulla base di una valutazione interpretativa (resa in assenza di norme codificate dal regolamento biologico) della compatibilità della norma tecnica di portata generale sull'abbattimento degli animali di cui al reg. 1099/09 (e non anche delle sue eccezioni), con i principi del regolamento sulla produzione biologica, negando in sostanza che quest'ultimo possa essere "corretto" da eccezioni che non garantirebbero, forse anche in ragione della loro indeterminatezza, il rispetto del principio della protezione del benessere animale.

Si esclude, così però anche, in radice, la compatibilità di una etichettatura biologica con le regole previste dai dettami religiosi ebraici o islamici: con la conseguenza, nelle intenzioni, di garantire il benessere animale (durante la fase dell'abbattimento), ma con l'ulteriore effetto, significativo sul piano delle scelte politiche, di rendere "incomunicabili" due segmenti dello stesso mercato, e di impedire - sul piano sociale e economico - l'integrazione tra due tipologie di consumatori.

Una scelta che, sia pure in un settore che potrebbe essere considerato "di nicchia”, quale quello delle produzioni di carni biologiche/halal/kasher, si riflette però su opzioni di fondo della normativa europea, in un confronto tra il "dettagliatamente normato" a livello europeo (quale è il settore biologico) e un ambito affidato invece a regole non codificate (i dettami religiosi per la fabbricazione del cibo). 